\title{
FLUIDODINÂMICA DE SEMENTES DE BRÓCOLOS EM LEITO FLUIDIZADO E LEITO DE JORRO
}

\author{
Celina de Almeida ${ }^{1,2}$; Sandra Cristina dos Santos Rocha ${ }^{1 *}$ \\ ${ }^{1}$ Depto. de Termofluidodinâmica - FEQ/UNICAMP, C.P. 6066 - CEP: 13083-970 - Campinas, SP. \\ ${ }^{2}$ Bolsista CAPES. \\ *Autor correspondente <rocha@feq.unicamp.br>
}

\begin{abstract}
RESUMO: Os processos em leito fluidizado e de jorro são comumente empregados para secagem, recobrimento e granulação de sistemas particulados nas indústrias química e farmacêutica, enquanto na área agronômica estão limitados a tratamento de algumas espécies de sementes. Este trabalho objetivou apresentar a análise fluidodinâmica dos leitos fluidizado e de jorro, quando são utilizadas sementes de brócolos (Brassica oleracea L. var. Italica) e verificar a influência na germinação das sementes, submetidas aos dois tipos de processo por 60 min à temperatura do ar de $25^{\circ} \mathrm{C}$. A fluidodinâmica nos dois tipos de contato, leitos fluidizado e de jorro, foi estabelecida pelas medidas da queda de pressão no leito e vazões de ar crescentes e decrescentes, para diferentes cargas de sementes. As condições experimentais foram baseadas nas análises das propriedades físicas das sementes e limitadas às dimensões do equipamento de laboratório. O leito cone-cilíndrico foi projetado e construído em acrílico, para permitir a visualização do processo. Os valores de queda de pressão máxima, vazão de jorro mínimo, vazão de jorro estável e queda de pressão no mínimo jorro foram obtidos a partir dos gráficos de queda de pressão-vazão para o leito de jorro. Estes valores foram comparados aos correspondentes valores obtidos por equações empíricas citadas na literatura. O mesmo procedimento foi realizado para o leito fluidizado obtendo-se os valores para velocidade de mínima fluidização e queda de pressão na mínima fluidização. Não houve danos à germinação das sementes que participaram dos processos.
\end{abstract}

Palavras-chave: queda de pressão, vazão, germinação, fluidização

\section{FLUID-DYNAMICS OF BROCCOLI SEEDS IN FLUIDIZED AND SPOUTED BEDS}

\begin{abstract}
The spouted and fluidized bed technologies are usually employed in operations of drying, coating and granulation of particles by the chemical and pharmaceutical industries. The use of these techniques in agronomy is limited to the treatment of some species of seeds. In this work, the objective was to analyse the fluid-dynamics of fluidized and spouted beds when broccoli (Brassica oleracea L. var. Italica) seeds are used and also to verify the influence on seed germination after $60 \mathrm{~min}$ of seed exposition to spouting or fluidization, at room temperature. The fluid-dynamics was defined by the measurements of the bed pressure drop as a function of the air flow rate for different seeds loads. The experimental conditions were based on the physical properties of the seeds and were limited by the apparatus dimensions. The cone-cylindrical bed was constructed in plexyglass to permit flow visualization. The values of the parameters: maximum pressure drop, minimum spouting flow rate and pressure drop, and stable spout pressure drop were experimentally obtained from the fluid-dynamic analysis and were compared with the values calculated by empirical equations found in the literature. The same procedure was carried out with the fluidized bed and the important parameters for this regime were the air velocity and the bed pressure drop at minimum fluidization. The analysis of seed germination indicated that no damage was caused to the seeds by the spout or fluidization processes.

Key words: pressure drop, flow rate, seed, germination, fluidization
\end{abstract}

\section{INTRODUÇÃO}

O estudo fluidodinâmico de partículas em leitos de jorro e fluidizado encontra inúmeras aplicações na indústria química, farmacêutica e recentemente, na área agrícola. A necessidade do aumento da produtividade agrícola tem contribuído para o desenvolvimento de novos projetos envolvendo sementes, com objetivo de promover condições favoráveis às sementes, para um possível armazenamento ou para a germinação imediata. Sendo assim, a otimização do processo e a minimização no custo de produção final da semente devem ser bem definidos (Taylor \& Harman, 1990).
A utilização da técnica de fluidização foi difundida em 1940, com a construção do primeiro leito fluidizado para craqueamento de carvão. Somente mais tarde esta técnica seria utilizada como meio adequado ao recobrimento de partículas. Vários estudos do desenvolvimento deste e de outros processos em leito fluidizado são apresentados por Kunni \& Levenpiel (1991). Nos anos 70, foi patenteado o processo para produção de sementes recobertas e métodos de recobrimento de sementes em leito fluidizado (Porter, 1974; Kitamura \& Watanabe, 1981; apud Kunii \& Levenspiel, 1991). Gishler \& Mathur (1957) obtiveram, para determinada faixa de tamanho de partículas, 0 
regime fluidodinâmico denominado de jorro, controlando a velocidade de entrada de gás e a forma geométrica do leito utilizado. Em 1962, no Canadá, foram instaladas as primeiras unidades de leito de jorro para secagem de ervilha, lentilha e sementes de linho. Recentemente, foi verificada a possibilidade de utilização do leito de jorro e leito fluidizado em alguns processos de revestimento de sementes, além dos processos de secagem, comumente utilizados.

A análise fluidodinâmica em leito fluidizado e de jorro com sementes tem contribuído na determinação dos valores de queda de pressão e vazão de fluido, característicos dos regimes de fluidização e do jorro, promovendo a otimização do custo através do adequado dimensionamento de equipamentos, juntamente com a determinação da energia necessária para o processo. Nesse contexto, é limitada a quantidade de trabalhos envolvendo sementes de hortaliças.

Drew (1987) analisou a germinação de sementes de Brassica napus L. após serem submetidas à secagem em leito fluidizado com variações no tempo de permanência das sementes no leito e na temperatura do processo. Concluiu que a produção de plântulas normais na germinação foi reduzida com o aumento da duração de fluidização juntamente com 0 aumento da temperatura. Além disso, o aumento no tempo de fluidização causou um aumento inicial no tempo médio de germinação.

O brócolos é cultivado no Brasil nas regiões sul e sudeste, em estações com temperaturas entre 15 e $20^{\circ} \mathrm{C}$. Na região centro oeste sua semeadura é recomendada para as estações do outono e inverno. A sensibilidade das sementes de brócolos às variações de temperatura e umidade relativa é responsável pelas variações na duração da colheita e diminuição da produção (Wurr et al., 1991 e 1992). Além disso, promovem diferença nos estádios de maturidade das sementes, o que foi verificado em análises de vigor por Lewis \& Welbaum (1996).

Os objetivos principais deste trabalho foram determinar o comportamento fluidodinâmico característico da fluidização e do jorro para diferentes cargas de sementes de brócolos; obter o valor da carga de sementes que melhor representa o comportamento fluidodinâmico para os leitos fluidizado e de jorro; definir experimentalmente para o leito de jorro, os valores dos parâmetros queda de pressão máxima no leito de jorro, velocidade de mínimo jorro, queda de pressão no jorro estável e queda de pressão no mínimo jorro; definir experimentalmente para o leito fluidizado os valores dos parâmetros queda de pressão na mínima fluidização e velocidade de mínima fluidização; definir as melhores correlações empíricas da literatura para estimativa dos parâmetros obtidos experimentalmente, calculando os respectivos desvios relativos; analisar a ocorrência de danos à germinação das sementes de brócolos, quando jorradas ou fluidizadas a $25^{\circ} \mathrm{C}$, durante $60 \mathrm{~min}$.

\section{MATERIAL E MÉTODOS}

As sementes de brócolos do cultivar Ramoso de Brasília foram obtidas da Empresa Brasileira de Pesquisa Agropecuária, lote $501 \mathrm{~A}$. A qualidade foi determinada para as sementes recém chegadas, através do teste de germinação, obtendo-se $93,71 \%$ de sementes germinadas; da análise de pureza, que constatou $99,56 \%$ de sementes puras; do peso de 1000 sementes, gerando um resultado de $4,2 \mathrm{~g}$ com coeficiente de variação de $2,37 \%$ e também da verificação do conteúdo de umidade, que ficou em $6,02 \%$ b. u. As propriedades físicas das sementes foram também determinadas: diâmetro médio de Sauter igual a $0,0017 \mathrm{~m}$, densidade real $1080 \mathrm{~kg} \mathrm{~m}^{-3}$, porosidade média do leito fixo de sementes de 0,34 e esfericidade igual a 0,48 .

O leito cone-cilíndrico foi usado para jorrar e fluidizar as sementes, sendo que para a utilização como jorro as sementes preencheram a parte cônica e 0,005 a $0,015 \mathrm{~m}$ da região cilíndrica. Para a utilização do equipamento como leito fluidizado foi colocado um distribuidor na flange, separando o cone da parte cilíndrica do leito. As sementes foram colocadas acima do distribuidor, preenchendo apenas parte do corpo cilindrico do leito.

A temperatura do ar foi mantida em torno de $25^{\circ} \mathrm{C}$ e monitorada através de três termopares (Figura 1).

O sistema operacional utilizado apresenta escala de laboratório, sendo assim, a escolha das cargas de sementes estavam limitadas às dimensões do leito. As sementes apresentaram características físicas enquadradas no grupo D de Geldart apud Kunii \& Levenspiel (1991), grupo das partículas facilmente jorráveis. A altura de semente em leito fixo $\left(\mathrm{H}_{0}\right)$, foi determinado em função da configuração de leito (Tabelas 1 e 2).

\section{Caracterização Experimental da Fluidodinâmica}

Os valores da vazão do ar de entrada no leito foram obtidos pelas medidas de pressão estática na linha em $\mathrm{mm}$ de $\mathrm{Hg}$ e queda de pressão na placa de orifício, em $\mathrm{mm}$ de $\mathrm{CCl}_{4}$ (tetracloreto de carbono), cujos valores inseridos na equação de calibração (1) permitem a obtenção da vazão mássica do $\operatorname{ar}(Q)$ em kg $\min ^{-1}$ (Ower \& Pankhurst, 1977).

$\mathrm{Q}=13,4255 \cdot\left(\frac{\Delta \mathrm{P}}{\mathrm{T}+273,15}\right)^{0,5}-\left[\frac{5,1781}{\mathrm{P}} \frac{(\Delta \mathrm{P})^{1,5}}{(\mathrm{~T}+273,15)^{0,5}}\right]$

em que: $\Delta P$ é a queda de pressão na placa de orifício em $\mathrm{cm}$ de $\mathrm{H}_{2} \mathrm{O}, \mathrm{P}$ a pressão estática na linha de ar em $\mathrm{g} \mathrm{cm}^{-1} \mathrm{~s}^{-1}$ e T a temperatura do ar em ${ }^{\circ} \mathrm{C}$.

Os valores experimentais da velocidade do ar foram calculados dividindo-se o valor da vazão volumétrica de ar pela área de entrada do leito. 


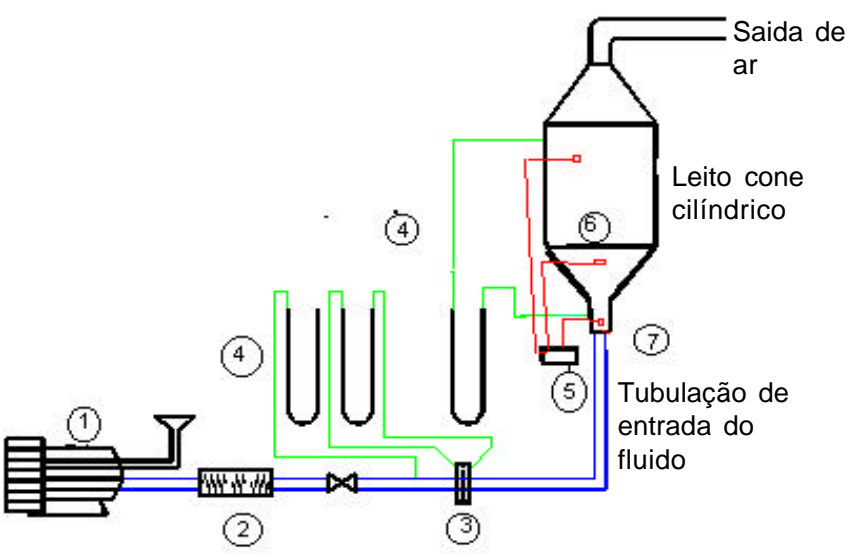

1 - soprador

2 - resfriador de ar

3 - placa de orifício

4 - manômetro em U

5 - termopares

6 - local para instalação da placa distribuidora para o leito fluidizado

7 - local para instalação do leito de jorro

$\mathrm{Dc}=0,20 \mathrm{~m}$ (diâmetro interno do leito)

$\theta=60^{\circ}$ (ângulo do cone)

$\mathrm{Di}=0,05 \mathrm{~m}$ (diâmetro da tubulação de entrada do fluido)

$\mathrm{H}_{0}=0,30 \mathrm{~m}$ (altura do leito estático)

$\mathrm{H}_{\mathrm{c}}=0,070 \mathrm{~m}$ (altura da parte cônica)

Figura 1 - Representação do sistema experimental.

Tabela 1 - Alturas das cinco cargas de sementes no leito para fluidização.

\begin{tabular}{lc}
\hline Carga de sementes & $\begin{array}{c}\text { Altura de sementes } \\
\text { no leito fixo }-\mathrm{H}_{0}\end{array}$ \\
\hline $\mathrm{kg}$ & $\mathrm{m}$ \\
0,5 & 0,019 \\
0,8 & 0,035 \\
1,1 & 0,043 \\
1,4 & 0,060 \\
1,7 & 0,072 \\
\hline
\end{tabular}

Tabela 2 - Alturas das cinco cargas de sementes no leito para jorro.

\begin{tabular}{|c|c|c|}
\hline $\begin{array}{l}\text { Carga de } \\
\text { sementes }\end{array}$ & $\begin{array}{c}\text { Altura de sementes } \\
\text { no cone }-\mathrm{H}_{c}\end{array}$ & $\begin{array}{c}\text { Altura total de } \\
\text { sementes no leito }-\mathrm{H}_{0}\end{array}$ \\
\hline $\mathrm{kg}$ & \multicolumn{2}{|c|}{ - } \\
\hline 0,5 & 0,055 & 0,055 \\
\hline 0,7 & 0,065 & 0,065 \\
\hline 0,9 & 0,070 & 0,070 \\
\hline 1,1 & 0,070 & 0,075 \\
\hline 1,3 & 0,070 & 0,085 \\
\hline
\end{tabular}

A queda de pressão no leito foi obtida em $\mathrm{mm}$ de água. Os valores de queda de pressão no leito correspondentes a cada valor de vazão de ar de entrada foram colocados em um gráfico, obtendo-se assim a curva que é denominada curva fluidodinâmica característica do leito.
O estudo fluidodinâmico foi realizado com cinco cargas diferentes de sementes de brócolos: 0,5 ; 0,$8 ; 1,1 ; 1,4$ e $1,7 \mathrm{~kg}$ para o leito fluidizado e 0,5 ; 0,$7 ; 0,9 ; 1,1$ e $1,3 \mathrm{~kg}$ para o leito de jorro. Foram construídas as curvas fluidodinâmicas características para todas as cargas de sementes dos leitos fluidizado e de jorro.

\section{Expressões que Definem Parâmetros no Processo de Fluidização}

A velocidade de mínima fluidização é um parâmetro importante, pois caracteriza o início do regime de fluidização no leito. Na prática, é comum operar um leito fluidizado com velocidades de duas a três vezes a de mínima fluidização. A queda de pressão por unidade de comprimento do leito permanece constante para velocidades acima da velocidade de mínima fluidização para um regime fluidodinâmico estável e fluidização de boa qualidade.

A previsão da velocidade de mínima fluidização foi obtida pela modificação da equação de Ergun apud Kunii \& Levenspiel (1991), a qual foi simplificada por Chitester et al. apud Kunii \& Levenspiel (1991).

$\frac{d_{p} \cdot U_{m f} \cdot \rho_{g}}{\mu_{g}}=\left[28,7^{2}+0,0494\left(\frac{d_{p}^{3} \cdot \rho_{g}\left(\rho_{r}-\rho_{g}\right) \cdot g}{\mu_{g}^{2}}\right)\right]^{0,5}-28,7$

em que: $\bigcup_{m f}$ é a velocidade de mínima fluidização em m $\mathrm{s}^{-1}, \mu_{\mathrm{g}}$ é a viscosidade do gás em $\mathrm{kg} \mathrm{m}^{-1} \mathrm{~s}^{-1}, \mathrm{~g} \mathrm{a}$ aceleração da gravidade e $\rho_{\mathrm{g}}$ a densidade do gás em $\mathrm{kg}$ $\mathrm{m}^{-3}$.

A queda de pressão na mínima fluidização $\left(\Delta \mathrm{P}_{\mathrm{mf}}\right)$ foi calculada pela equação (3), baseada no princípio da fluidização, em que a força de arraste das partículas se iguala ao peso dessas partículas no ponto de mínima fluidização.

$$
\Delta \mathrm{P}_{\mathrm{mf}}=\mathrm{L}_{\mathrm{mf}}\left(1-\varepsilon_{\mathrm{mf}}\right) \cdot\left(\rho_{\mathrm{r}}-\rho_{\mathrm{g}}\right) \mathrm{g}
$$

em que: $L_{m f}$ é a altura do leito na mínima fluidização em $\mathrm{m}$ e $\varepsilon_{\mathrm{mt}}$ a porosidade na mínima fluidização

\section{Expressões que Definem Parâmetros no Processo de Jorro}

Os parâmetros utilizados são: queda de pressão máxima no leito de jorro $\left(\Delta \mathrm{P}_{\mathrm{m}}\right)$ - que define, juntamente com a vazão correspondente, a máxima energia mecânica necessária para o sistema jorrar; velocidade de mínimo jorro $\left(\mathrm{U}_{\mathrm{ms}}\right)$ - velocidade acima da qual ocorre o regime fluidodinâmico de jorro, correspondendo a $U_{m t}$ para a fluidização; queda de pressão no jorro estável $\left(\Delta \mathrm{P}_{\mathrm{s}}\right)$ - praticamente constante para velocidade de operação acima do jorro mínimo; queda de pressão no mínimo jorro $\left(\Delta \mathrm{P}_{\mathrm{ms}}\right)-$ queda de pressão para $\mathrm{U}=\mathrm{U}_{\mathrm{ms}}$. Os parâmetros descritos estão representados pelas equações nas Tabelas 3, 4, 5 e 6, e apresentam condições de contorno, que se enquadraram às condições experimentais deste trabalho. 
Foram feitas as comparações entre os valores dos parâmetros experimentais e valores resultantes das equações teóricas, sendo representadas por desvios relativos, calculados da seguinte maneira:

Desvio relativo $=\mid$ valor teórico - valor experimental $\mid$. 100 / valor experimental (19)

\section{Teste de Germinação}

Uma amostra de trabalho contendo 400 sementes foi retirada ao acaso dos lotes de sementes sem tratamento e das submetidas aos regimes de jorro e fluidizado. Com estas sementes realizou-se o teste de germinação imediatamente após os ensaios. Cada amostra foi colocada em quatro placas com 100 sementes. As placas "gerbox" continham papel especial "germitest", embebido em água destilada com cerca de 2,5 vezes a sua massa e o espaçamento entre as sementes foi uniforme, para minimizar a competição e contaminação.

Tabela 3 - Equação para velocidade de mínimo jorro $\left(U_{m s}\right)$.

\begin{tabular}{lll}
\hline Autor & Equação & Condições de contorno \\
\hline Tsvik et al. (1967) & $\left(\operatorname{Re}_{\mathrm{i}}\right)_{\mathrm{ms}}=0,4(\mathrm{Ar})^{0,52}\left(\frac{\mathrm{H}_{0}}{\mathrm{D}_{\mathrm{i}}}\right)^{1,24}\left(\tan \frac{\theta}{2}\right)^{0,42}=\frac{\left.\mathrm{U}_{\mathrm{ms}}\right)_{\mathrm{i}} \rho g \mathrm{gd}_{\mathrm{p}}}{\mu \mathrm{g}}$ & $\begin{array}{l}\text { Re }=\text { número de Reyno lds } \\
\mathrm{D}_{\mathrm{i}}=2,0 \mathrm{a} 4,2 \mathrm{~cm}\end{array}$ \\
& $\begin{array}{l}\mathrm{H}_{\mathrm{o}}=10-50 \mathrm{~cm} \\
\mathrm{~d}_{\mathrm{p}}=1,5 \mathrm{a} 4,0 \mathrm{~mm} \\
\theta=20^{\circ} \mathrm{a} 50^{\circ}\end{array}$ \\
& $\begin{array}{l}\left.\rho_{\mathrm{b}}=780 \mathrm{a} 840 \mathrm{~kg} \mathrm{~m}\right)^{-3} \\
\rho_{\mathrm{r}}=1650 \mathrm{a} 1700 \mathrm{~kg} \mathrm{~m}^{-3}\end{array}$ \\
\hline
\end{tabular}

Tabela 4 - Equações para queda de pressão máxima no leito de jorro $\left(\Delta \mathrm{P}_{\mathrm{m}}\right)$.

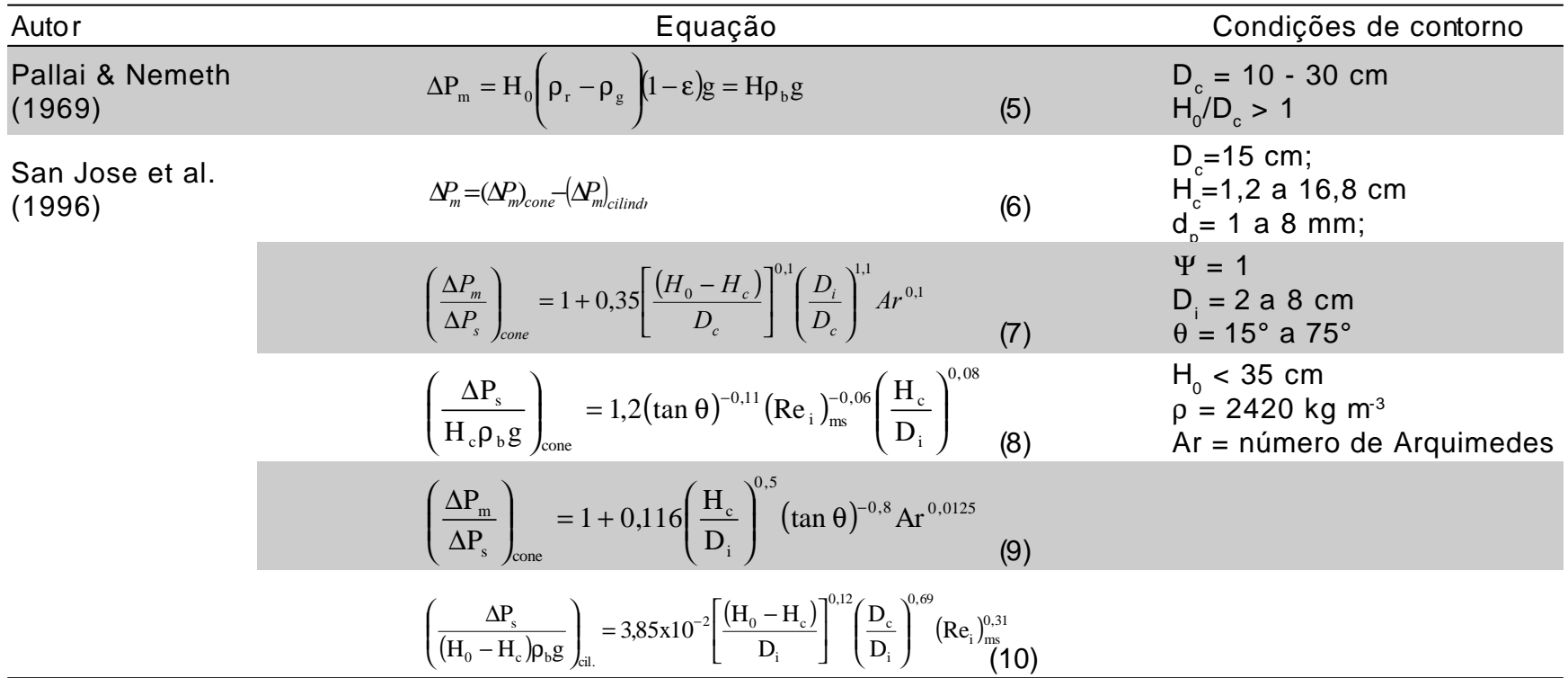

Tabela 5 - Equações para queda de pressão no jorro estável $\left(\mathrm{DP}_{\mathrm{s}}\right)$.

\begin{tabular}{|c|c|c|c|}
\hline Autor & Equação & & Condições de contorno \\
\hline $\begin{array}{l}\text { Pallai \& Nemeth } \\
\text { (1969) }\end{array}$ & $\frac{\left(\Delta \mathrm{P}_{\mathrm{s}}\right)}{\Delta \mathrm{P}_{\mathrm{m}}}=0,8-0,01 \frac{\mathrm{D}_{\mathrm{c}}}{\mathrm{D}_{\mathrm{i}}}$ & (11) & $D_{c}=15,2$ a $61 \mathrm{~cm}$ \\
\hline \multirow[t]{4}{*}{$\begin{array}{l}\text { San Jose et al. } \\
(1996)\end{array}$} & $\Delta P_{\mathrm{s}}=\left(\Delta \boldsymbol{P}_{\mathrm{s}}\right)_{\text {cone }}+\left(\Delta \boldsymbol{P}_{\mathrm{s}}\right)_{\text {cilindr }}$ & (12) & $\begin{array}{l}\mathrm{D}_{\mathrm{c}}=15 \mathrm{~cm} \\
\mathrm{H}_{\mathrm{c}}=1,2 \text { a } 16,8 \mathrm{~cm} \\
d_{\mathrm{p}}=1 \text { a } 8 \mathrm{~mm}\end{array}$ \\
\hline & $\left(\frac{\Delta P_{s}}{\left(H_{0}-H_{c}\right) \rho_{b} g}\right)_{\text {cil. }}=3,85 \times 10^{-2}\left[\frac{\left(H_{0}-H_{c}\right.}{D_{i}}\right]^{0,12}\left(\frac{D_{c}}{D_{i}}\right)^{0,69}\left(R_{i} e_{m s}^{0,31}\right.$ & (13) & $\begin{array}{l}\Psi=1 \\
D_{i}=2 \text { a } 8 \mathrm{~cm} \\
\theta=15^{\circ} \text { a } 75^{\circ}\end{array}$ \\
\hline & $\left(\frac{\Delta P_{m}}{\Delta P_{s}}\right)_{\text {cone }}=1+0,35\left[\frac{\left(H_{0}-H_{c}\right)}{D_{c}}\right]^{0,1}\left(\frac{D_{i}}{D_{c}}\right)^{1,1} A r^{0,1}$ & (14) & $\begin{array}{l}\mathrm{H}_{0}<35 \mathrm{~cm} \\
\rho=2420 \mathrm{~kg} \mathrm{~m}^{-3}\end{array}$ \\
\hline & $\left(\operatorname{Re}_{i}\right)_{m s}=0,126 A r^{0,5}\left(\frac{D_{c}}{D_{i}}\right)^{1,68}(\tan \theta)^{-0,57}$ & (15) & \\
\hline
\end{tabular}


As sementes foram mantidas em câmara de germinação por cinco dias em ambiente de alta umidade relativa, temperatura variando de 25 a $30^{\circ} \mathrm{C}$ e iluminação permanente. Após este período, as sementes que não germinaram retornaram à câmara por mais cinco dias e sofreram nova avaliação (ISTA, 1985).

Nas contagens, todas as plântulas e sementes não germinadas foram classificadas em: plântulas normais, plântulas anormais, sementes não germinadas e sementes multi germinadas (ISTA, 1985). As respostas da análise de germinação foram calculadas através da soma de plântulas normais e sementes duras por placa "gerbox", dadas em porcentagem.

Seguindo um delineamento inteiramente casualizado foi realizada a análise estatística dos resultados de germinação. Os tratamentos foram definidos em: sementes testemunha, sementes jorradas e sementes fluidizadas $A$ análise de variância foi utilizada para avaliar a diferença entre as médias dos tratamentos $\left(\mu_{i}\right)$, pelo teste $F$, para um nível de significância de $5 \%$ na distribuição de Fisher.

\section{RESULTADOS E DISCUSSÃO}

\section{Fluidodinâmica para leito fluidizado}

Com o aumento da velocidade de ar, chega-se a um ponto em que o leito fixo "expande", aumentando a porosidade inicial das sementes $(\varepsilon)$ para a porosidade de mínima fluidização $\left(\varepsilon_{\mathrm{mf}}\right)$. A partir desse ponto, definido como velocidade de mínima fluidização $\left(\cup_{m f}\right)$, o aumento na taxa de escoamento de ar causa pequenas variações na queda de pressão mínima fluidização $\left(\Delta \mathrm{P}_{\mathrm{m}}\right)$. Os parâmetros $U_{m f}, \varepsilon_{m f}$ e $\Delta P_{m f}$ são diretamente relacionados às propriedades do sólido e do gás e caracterizam o início do regime de fluidização, os quais possibilitam otimizar o processo e dimensionar os equipamentos e soprador.

A curva para o leito fixo apresentou inicialmente um comportamento quadrático, devido ao leito fluidizar somente após alcançar altas vazões. Esta parte da curva foi ajustada a um polinômio de segundo grau.

As curvas fluidodinâmicas, para todas as cargas de sementes em leito fluidizado, apresentaram características semelhantes bem evidentes, entretanto, após o início da fluidização, a curva obtida para a carga de $1,4 \mathrm{~kg}$ apresentou menores variações de queda de pressão no leito, facilitando a visualização do comportamento fluidodinâmico. Este foi o motivo de escolhermos apenas esta curva de fluidização a ser representada neste trabalho (Figura 2).

O valor experimental da queda de pressão na mínima fluidização corresponde à ordenada do ponto definido como de mínima fluidização, a partir do qual a queda de pressão permanece praticamente constante. A abscissa é a vazão de mínima fluidização experimental.

As previsões teóricas estão próximas dos valores experimentais (Tabela 7). As correlações que apresentaram desvio relativo inferior a $20 \%$ são consideradas satisfatórias, segundo Kunii \& Levenspiel (1991), para a estimativa desses parâmetros. Os desvios relativos para velocidade de mínima fluidização podem

Tabela 6 - Equações para queda de pressão no mínimo jorro $\left(\Delta \mathrm{P}_{\mathrm{ms}}\right)$.

\begin{tabular}{|c|c|c|c|}
\hline Autor & Equação & & Condições de contorno \\
\hline $\begin{array}{l}\text { Malek \& Lu } \\
\text { (1965) apud } \\
\text { Massarani } \\
\text { (1987) }\end{array}$ & $\Delta P_{m s}=\frac{2}{3} \frac{M g}{A_{c i l}}$ & (16) & $\begin{array}{l}d_{p}=4 \text { a } 6 \mathrm{~mm} \\
\theta=60^{\circ} \\
\rho=1110 \text { a } 1190 \mathrm{~kg} \mathrm{~m}^{-3} \\
\Psi=0,8 \text { a } 0,9 \\
A_{d i}=\text { área da seção cilíndrica do leito } \\
M=\text { a massa de partículas no leito }\end{array}$ \\
\hline $\begin{array}{l}\text { Nascimento et } \\
\text { al. apud } \\
\text { Massarani } \\
(1987)\end{array}$ & $\Delta \mathrm{P}_{\mathrm{ms}}=0,67\left(1-\varepsilon_{\mathrm{mf}}\right) \mathrm{H}_{0} \rho_{\mathrm{r}} \mathrm{g}$ & (17) & $\begin{array}{l}d_{p}=4 \text { a } 6 \mathrm{~mm} \\
\theta=60^{\circ} \\
\rho=1110 \text { a } 1190 \mathrm{~kg} \mathrm{~m}^{-3} \\
\Psi=0,8 \text { a } 0,9\end{array}$ \\
\hline $\begin{array}{l}\text { Sampaio } \\
(1978)\end{array}$ & $\frac{\Delta \mathrm{P}_{\mathrm{ms}}}{\rho_{\mathrm{b}} \mathrm{gH}{ }_{0}}=\frac{2}{3}$ & (18) & $\begin{array}{l}d_{p}=4 \text { a } 6 \mathrm{~mm} \\
\theta=60^{\circ} \\
\rho=1110 \text { a } 1190 \mathrm{~kg} \mathrm{~m}^{-3} \\
\Psi=0,8 \text { a } 0,9\end{array}$ \\
\hline
\end{tabular}

Tabela 7 - Resultados da velocidade de mínima fluidização $\left(U_{m t}\right)$ e queda de pressão no leito fluidizado $(\Delta P)$.

\begin{tabular}{|c|c|c|c|c|c|c|}
\hline Carga de semente & $\mathrm{U}_{\mathrm{mf}}$ experimental & $U_{m f}$ eq. (2) & Desvio relativo & $\Delta \mathrm{P}$ experimental & $\Delta \mathrm{P}$ eq. (3) & Desvio relativo \\
\hline $\mathrm{kg}$ & -------- $\mathrm{m} \mathrm{s}^{-1}$ & ---------- & ------ \% ------- & --------- Pa & --------- & ----- \% - \\
\hline 0,5 & 0,607 & 0,643 & 5,9 & 190 & 132,01 & 30,5 \\
\hline 0,8 & 0,579 & 0,643 & 11,1 & 270 & 243,18 & 9,9 \\
\hline 1,1 & 0,576 & 0,643 & 11,6 & 370 & 298,76 & 19,3 \\
\hline 1,4 & 0,560 & 0,643 & 14,8 & 440 & 416,87 & 5,3 \\
\hline 1,7 & 0,539 & 0,643 & 19,3 & 530 & 500,25 & 5,6 \\
\hline
\end{tabular}


ser explicados pela Equação (3) estar sendo utilizada em forma simplificada. Descarte-se a correlação de queda de pressão na mínima fluidização para a carga de 0,5 $\mathrm{kg}$, que apresentou desvios acima de $20 \%$, devido à dificuldade encontrada para se medir a altura das sementes no leito fixo.

\section{Fluidodinâmica para leito de jorro}

A queda de pressão máxima correspondente a $732 \mathrm{~Pa}$ ocorreu exatamente antes de haver $\mathrm{o}$ estabelecimento do jorro, quando a vazão de ar atingiu aproximadamente $0,34 \mathrm{~kg} \mathrm{~min}^{-1}$, correspondente à velocidade de mínimo jorro (Figura 3). Esta é a velocidade do ar que possibilita o início do movimento de jorro.

Com o aumento contínuo da vazão chegamos à queda de pressão de jorro estável, onde também pôdese observar visualmente o estabelecimento do regime de jorro. Os valores de vazão em jorro estável ficaram na faixa de 0,5 a $0,67 \mathrm{~kg} \mathrm{~min}^{-1}$, sendo considerada uma faixa pequena, comparada a faixas comumente verificadas em literatura. Após a vazão de $0,67 \mathrm{~kg} \mathrm{~min}^{-1}$ foi visível o comportamento de jorro rápido, com aumento expressivo da altura da fonte.

Com o decréscimo da vazão, após a velocidade de jorro estável, chegou-se ao ponto de queda de

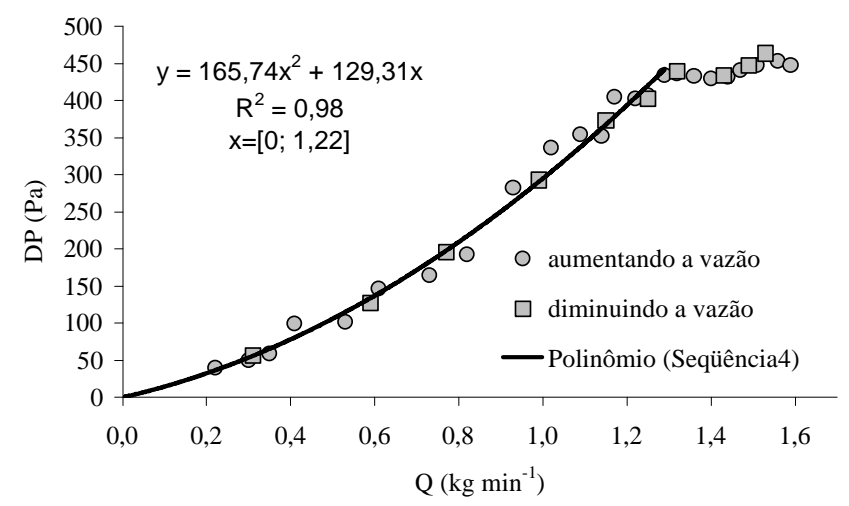

Figura 2 - Queda de pressão, em função da vazão para leito fluidizado, com carga de brócolos de 1,4 kg.

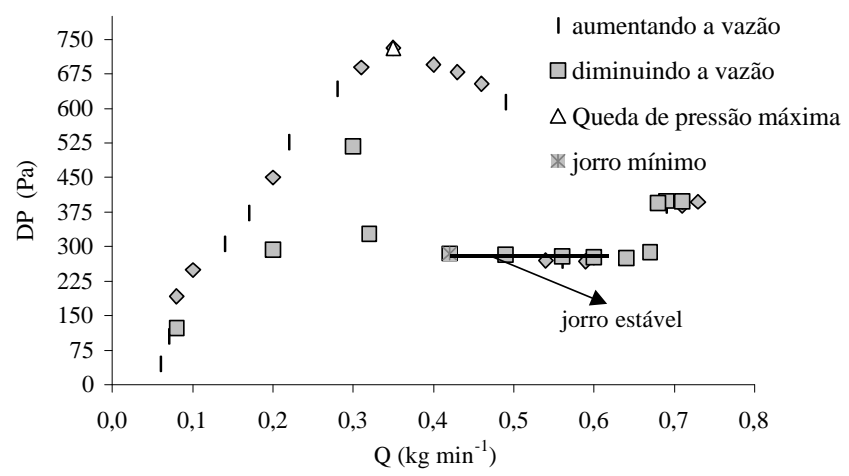

Figura 3 - Queda de pressão, em função da vazão para leito de jorro com, carga de brócolos de $0,9 \mathrm{~kg}$. pressão do jorro mínimo, que na curva corresponde à vazão de $0,42 \mathrm{~kg} \mathrm{~min}^{-1}$ e pressão de $285 \mathrm{~Pa}$. A partir deste ponto o decréscimo da vazão causou o colapso do jorro.

Todas as curvas fluidodinâmicas para o leito de jorro, envolvendo as cinco cargas de sementes, foram semelhantes e apresentaram os pontos característicos de queda de pressão máxima, jorro estável, jorro rápido, e jorro mínimo. Entretanto, a curva obtida para carga de $0,9 \mathrm{~kg}$ possibilitou a melhor definição do regime e dos parâmetros. Sendo assim, é apresentada apenas esta curva do leito de jorro.

\section{Velocidade de jorro mínimo $\left(U_{m s}\right)$}

A equação proposta por Tsvik et al. (1967) apresentou desvio relativo aceitável para velocidade de jorro mínimo para a carga de $0,5 \mathrm{~kg}$, estando o desvio relativo abaixo de $20 \%$ (Tabela 8 ). Como também nesse caso, a velocidade de operação é sempre bem maior que a de jorro mínimo, desvios relativos da ordem de $20 \%$ são considerados aceitáveis (Mathur \& Epstein, 1974). Existe uma limitação na precisão da determinação experimental da vazão de jorro mínimo, podendo incorrer em maiores desvios quando da comparação com valores obtidos de equações empíricas. $O$ valor experimental da velocidade de jorro mínimo é determinado a partir do estudo fluidodinâmico, para vazões decrescentes, no ponto onde ocorre o colapso do jorro. Esse ponto não tem uma identificação precisa no diagrama fluidodinâmico, sendo, portanto de difícil reprodução. A dificuldade na determinação deste parâmetro experimentalmente pode ter como conseqüência a obtenção de altos desvios relativos entre medidas efetuadas por diferentes pesquisadores, bem como entre resultados experimentais e calculados.

\section{Queda de pressão máxima $\left(\Delta \mathbf{P}_{\mathrm{m}}\right)$}

Foi possível obter boa concordância entre os valores experimentais e teóricos para a queda de pressão máxima no leito de jorro (Tabela 9).

As equações que forneceram as melhores previsões foram de San Jose et al. (1996) e Pallai \& Nemeth (1969), representadas pelas equações (6) e (5). As demais correlações obtidas na literatura foram descartadas por apresentar desvio relativo acima de $20 \%$. A correlação de San Jose et al. (1996) foi a que apresentou os melhores resultados para todas as cargas

\begin{tabular}{|c|c|c|c|}
\hline $\begin{array}{l}\text { Carga de } \\
\text { sementes }\end{array}$ & $\begin{array}{c}\cup_{\mathrm{ms}} \\
\text { Experimental }\end{array}$ & $\begin{array}{c}U_{m s} \\
\text { Eq. (4) }\end{array}$ & $\begin{array}{l}\text { Desvio } \\
\text { relativo }\end{array}$ \\
\hline $\mathrm{kg}$ & \multicolumn{2}{|c|}{--------- m s$^{-1}$-------- } & ------ \% ------ \\
\hline 0,5 & 1,79 & 1,54 & 14,0 \\
\hline 0,7 & 2,52 & 1,90 & 24,5 \\
\hline 0,9 & 3,02 & 2,27 & 24,9 \\
\hline 1,1 & 3,59 & 2,65 & 26,2 \\
\hline 1,3 & 3,82 & 3,04 & 20,3 \\
\hline
\end{tabular}


de sementes. A correlação de Pallai \& Nemeth (1969) apresentou melhores resultados para as cargas de 0,7 e $0,9 \mathrm{~kg}$. Não existe uma justificativa física para a diferença de comportamento das correlações testadas, já que as condições de contorno experimentais são satisfeitas para todas essas correlações. Entretanto, resultados desse tipo não são incomuns em se tratando de análises empíricas, as quais envolvem tanto similaridades geométrica e dinâmica entre os sistemas, como também apresentam influência as faixas de propriedades físicas dos materiais utilizados nos experimentos e a precisão na obtenção dos parâmetros experimentais.

\section{Queda de pressão no jorro estável $\left(\Delta \mathrm{P}_{\mathrm{s}}\right)$}

Para a queda de pressão estável no leito de jorro não foi encontrada nenhuma equação adequada na literatura, que fornecesse boa previsão para esse parâmetro (Tabela 10). Todas as correlações apresentaram desvios relativos acima de $70 \%$, o que é inaceitável, mesmo levando-se em conta a faixa estreita obtida para jorro estável com as sementes de brócolos. Verifica-se a ocorrência de pequena faixa de vazão para obtenção dos valores experimentais de jorro estável (Figura 3), e consideramos ser essa a causa dos grandes desvios entre os valores experimentais e calculados pelas correlações.

\section{Queda de pressão no jorro mínimo $\left(\Delta \mathrm{P}_{\mathrm{ms}}\right)$}

Baseando-se no desvio relativo inferior a $20 \%$, como limite aceitável, a equação de Nascimento et al. apud Massarani (1987) forneceu os melhores resultados para $\Delta \mathrm{P}_{\text {ms }}$, com desvio entre 5,7 e $21 \%$ (Tabela 11). A correlação de Sampaio (1978) apresentou desvios acima de $30,9 \%$ para as maiores cargas, e resultado aceitável apenas para a carga de 0,5 kg. Já a correlação de Malek \& Lu (1965) apresentou resultado aceitável apenas para a carga de 1,3 kg. Os altos desvios relativos para as correlações que estimaram os parâmetros mostraram que estas são limitadas, principalmente quando se trabalha com condições experimentais pouco amplas.

\section{Análises das sementes de brócolos}

$\mathrm{O}$ atrito semente-semente e semente-parede decorrente da exposição das sementes a movimentos cíclicos dentro do leito por $60 \mathrm{~min}$, causou danos pouco evidentes de fissura e quebras, sendo que menos de $1 \%$ das sementes fluidizadas apresentaram parte do seu tegumento quebrado.

Foram preservadas as estruturas da semente após terem sido submetidas às condições experimentais, devido à grande resistência a choques mecânicos que estas sementes apresentam, favorecida pela sua característica física. Drew (1987) obteve bons resultados em relação a danos físicos quando utilizou a fluidização por 60 min a temperatura de $25^{\circ} \mathrm{C}$, com sementes de Brassica napus L.

A permanência das sementes de brócolos em fluidização e jorro causou variação no teor de umidade, com queda de $6,02 \%$ para $5,75 \%$ b.u. nas sementes jorradas e de $6,02 \%$ para $5,67 \%$ b.u. nas sementes fluidizadas. Drew (1987) também verificou a queda de umidade para as sementes de Brassica napus L. fluidizadas em temperatura de $25^{\circ} \mathrm{C}$, sendo os valores semelhantes ao encontrados neste trabalho. A diminuição da umidade pode ser um problema para a semente quando ela atinge um valor muito baixo, aumentando a susceptibilidade a danos mecânicos.

O lote de sementes de brócolos apresentou germinação inicial de $93,75 \%$ (Tabela 12). Comparandose com o valor mínimo de germinação de $80 \%$, estipulado pelo Ministério da Agricultura de São Paulo para o comercio de sementes de brócolos, considera-se o lote de sementes utilizado nos experimentos de alto valor germinativo, assegurando os resultados obtidos neste trabalho.

Tabela 9 - Resultados para queda de pressão máxima ( $\mathrm{DP}_{\mathrm{m}}$ ).

\begin{tabular}{|c|c|c|c|c|c|}
\hline Carga de sementes & $\Delta \mathrm{P}_{\mathrm{m}}$ Experimental & $\Delta \mathrm{P}_{\mathrm{m}}$ Eq. (6) & Desvio relativo & $\mathrm{DP}_{\mathrm{m}}$ Eq.(5) & Desvio relativo \\
\hline $\mathrm{kg}$ & 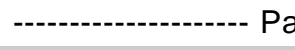 & 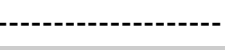 & -------- \% ------- & ------- Pa ------- & -------- \% -------- \\
\hline 0,5 & 505,68 & 448,52 & 11,3 & 355,87 & 29,6 \\
\hline 0,7 & 603,24 & 640,72 & 6,2 & 498,23 & 17,4 \\
\hline 0,9 & 732,00 & 731,45 & 0,1 & 590,74 & 19,3 \\
\hline 1,1 & 872,73 & 740,98 & 15,1 & 638,95 & 26,8 \\
\hline 1,3 & 881,26 & 769,48 & 12,7 & 692,02 & 21,5 \\
\hline
\end{tabular}

Tabela 10 - Resultados para queda de pressão estável $\left(\Delta \mathrm{P}_{\mathrm{s}}\right)$.

\begin{tabular}{|c|c|c|c|c|c|}
\hline Carga de sementes & $\Delta \mathrm{P}_{\mathrm{s}}$ Experimental & $\Delta \mathrm{P}_{\mathrm{s}}$ Eq. (12) & Desvio relativo & $\Delta \mathrm{P}_{\mathrm{s}}$ Eq.(11) & Desvio relativo \\
\hline $\mathrm{kg}$ & - - & 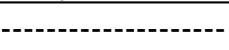 & ------- \% ------- & - & ------- \% ------- \\
\hline 0,5 & 204 & 384,64 & 70,9 & 384,32 & 88,4 \\
\hline 0,7 & 231 & 488,57 & 111,5 & 458,46 & 98,5 \\
\hline 0,9 & 269 & 554,64 & 106,2 & 556,32 & 106,8 \\
\hline 1,1 & 302 & 565,39 & 87,2 & 663,27 & 119,6 \\
\hline 1,3 & 292 & 590,41 & 102,2 & 669,76 & 129,4 \\
\hline
\end{tabular}


Tabela 11 - Resultados para queda de pressão no jorro mínimo $\left(\Delta \mathrm{P}_{\mathrm{ms}}\right)$.

\begin{tabular}{|c|c|c|c|c|c|c|c|}
\hline $\begin{array}{l}\text { Carga de } \\
\text { sementes }\end{array}$ & $\Delta \mathrm{P}_{\mathrm{ms}}$ Experimental & $\begin{array}{c}\Delta \mathrm{P}_{\mathrm{ms}} \\
\text { Eq. (17) }\end{array}$ & $\begin{array}{l}\text { Desvio } \\
\text { relativo }\end{array}$ & $\begin{array}{c}\Delta \mathrm{P}_{\mathrm{ms}} \\
\mathrm{Eq} \cdot(18)\end{array}$ & $\begin{array}{l}\text { Desvio } \\
\text { relativo }\end{array}$ & $\begin{array}{c}\Delta \mathrm{P}_{\mathrm{ms}} \\
\text { Eq.(16) }\end{array}$ & $\begin{array}{l}\text { Desvio } \\
\text { relativo }\end{array}$ \\
\hline $\mathrm{kg}$ & -------------- Pa -- & ----------- & $----\%$ & --- Pa ---- & $----\%$---- & ------ Pa ------- & -------- \% ------- \\
\hline 0,5 & 232,34 & 183,47 & 21,0 & 237,25 & 2,1 & 103,99 & 55,2 \\
\hline 0,7 & 253,79 & 216,82 & 14,6 & 332,15 & 30,9 & 145,58 & 42,6 \\
\hline 0,9 & 285,00 & 250,18 & 12,2 & 393,82 & 38,2 & 187,18 & 34,3 \\
\hline 1,1 & 300,67 & 283,54 & 5,7 & 425,96 & 41,7 & 228,77 & 23,9 \\
\hline 1,3 & 299,61 & 316,90 & 5,8 & 461,35 & 54,0 & 270,36 & 9,8 \\
\hline
\end{tabular}

Tabela 12 - Resultados do teste de germinação.

\begin{tabular}{|c|c|c|c|c|c|}
\hline Semente brócolo & $\begin{array}{c}\text { Plântula } \\
\text { normal }\end{array}$ & $\begin{array}{l}\text { Plântula } \\
\text { anormal }\end{array}$ & $\begin{array}{l}\text { Plântula } \\
\text { infectada }\end{array}$ & Semente dura & $\begin{array}{c}\text { Semente } \\
\text { morta }\end{array}$ \\
\hline & \multicolumn{5}{|c|}{ 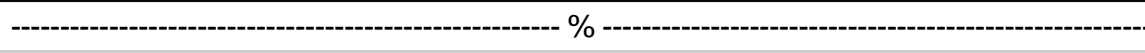 } \\
\hline Média para semente testemunha & 93,00 & 2,00 & 1,00 & 0,25 & 3,75 \\
\hline Desvio padrão & 0,82 & 0,82 & 0,82 & 0,50 & 1,26 \\
\hline Média para semente fluidizada & 91,00 & 4,00 & 1,25 & 0 & 3,75 \\
\hline Desvio padrão & 2,16 & 0,82 & 0,96 & -- & 1,26 \\
\hline Média para semente jorrada & 91,75 & 3,00 & 1,50 & 0 & 3,75 \\
\hline Desvio padrão & 2,22 & 0,82 & 1,29 & --- & 0,96 \\
\hline
\end{tabular}

Não existiu diferença entre os valores de germinação para os tratamentos. Verificou-se assim, a aptidão das sementes em produzir plântulas normais sob condições de laboratório.

\section{CONCLUSÕES}

É possível fluidizar e jorrar as sementes de brócolos, em escala de laboratório, sem causar danos às estruturas essenciais do embrião, possibilitando a normal emergência e desenvolvimento destas sementes. Sugerimos a realização do teste de vigor para futuros trabalhos envolvendo fluidização e jorro, nos quais será possível determinar também o potencial fisiológico das sementes.

\section{AGRADECIMENTOS}

Ao Prof. Dr. Luis Fernandes Razera, Instituto Agronômico de Campinas, Seção de Materiais Propagativos, pela colaboração para a realização deste trabalho.

\section{REFERÊNCIAS BIBLIOGRÁFICAS}

DREW, R.L.K. The effects of duration and temperature of treatment in a prototype fluidized-bed seed treatment on the subsequent germination of seed of oilseed rape Brassica napus L. Seed Science and Technology, v.17, p.7-13, 1987.

GISHLER, P.E.; MATHUR, K.B. Method of contacting solid particles with fluids. Ottawa: National Research Council of Canada, 1957. US 2.786.280; UK 801315.
INTERNATIONAL SEED TESTING ASSOCIATION (ISTA). International rules for seed testing. Seed Science and Technology, v.13, p.322-326, 1985.

KUNII, D.; LEVENSPIEL, O. Fluidization engineering. New York: John Wiley, 1991. 489p.

LEWIS, W.J.; WELBAUM, G.E. Changes in broccoli Brassica oleracea L. seed weight, viability, and vigor during development and following drying and priming. Seed Science and Technology, v.24, p.127-137, 1996.

MASSARANI G. Secagem de produtos agrícolas. Rio de Janeiro: Edgard Bücher, 1987. v.2, 56p.

MALEK, M.A.; LU, B.C.Y. Pressure drop and spoutable bed height in spouted bed. Industrial and Engineer Chemical Process Design and Develop, v.4, p.123-128, 1965.

MATHUR, K. B.; EPSTEIN, N. Spouted beds 1.ed. London: Acadmic Press, 1974. 304p.

OWER, E.; PANKHURST, R.C. The measurement of air flow. 5.ed. New York: Pergamon Press, 1977. p.51-54.

PALLAI, I.; NEMETH, J. Analysis of flow forms in a spouted bed apparatus by the so-called phase diagram. In: INTERNATIONAL CONGRESS CHEMICAL ENGINEER (CHISA), 3., Praga, 1969. Praga: Czechoslovak Society for Industrial Chemistry, 1969. C.2.4.

SAMPAIO, L.S.V. Dinâmica do leito de jorro. Rio de Janeiro, 1978. 120p. Dissertação (Mestrado) - Universidade Federal do Rio de Janeiro.

SAN JOSE, M.J.; OLAZAR, M.; AGUADO, R.E.; BILBAO, J. Influence of the conical section geometry on the hydrodynamics of shallow spouted. Chemical Engineer Journal, v.62, p.113-120, 1996.

TAYLOR, A.G.; HARMAN, G.E. Concepts and technologies of selected seed treatments, Phytopathology, v.28, p.321-339, 1990.

TSVIK, M.Z.; NABIEV, M.N.; RIZAEV, N.U.; MERENKOV, K.V.; VYZGO, V.S. The velocity for external spouting in the combined process for production of granulated fertilizers. Uzbekskii Khimichesk, v.11, p.50-69, 1967.

WURR, D.C.E.; FELLOWS, J.R.; HMBIDGE, A.J. The influence of field environmental conditions on Calabrese growth and development. Journal of Horticultural Science, v.66, p.495-504, 1991.

WURR, D.C.E.; FELLOWS, J.R.; HMBIDGE, A.J. The effect of plant density on Calabrese growth and its use in a predictive model. Journal of Horticultural Science, v.67, p.77-85, 1992.

Recebido em 26.04.01 